\title{
Mucinous Adenocarcinoma of Colon Presenting As Cutaneous Metastases over The Abdominal Wall: A Rare Entity on Cytology
}

\author{
Gireesha Rawal*, Mukul Singh, Charanjeet Ahluwalia and AK Mandal
}

Department of Pathology, Vardhman Mahavir Medical College and Safdarjung Hospital, New. Delhi. India

\begin{abstract}
Skin metastases from colorectal carcinoma are rare and typically signify widespread disease with poor prognosis. We present two cases of a 55 years old man and a 57 years old lady, both presenting with multiple skin nodules on the abdominal wall. FNAC smears of both the cases showed clusters and sheets of atypical cells with moderate amount of cytoplasm against a necrotic background. Nuclei were round to oval with coarse chromatin and prominent nucleoli. Well formed glandular structures were seen. Immunocytochemistry showed that cells were positive for Muc-1, and negative for vimentin and LCA. A diagnosis of adenocarcinoma presenting as cutaneous metastasis was given. Detailed history, showed that both the patients were known cases of adenocarcinoma colon post chemotherapy and radiotherapy. Colorectal carcinoma presenting as cutaneous metastases is rare and FNAC is a noninvasive and fast method for directing towards the diagnosis of cutaneous metastasis in the patients with a known malignancy without any complication.
\end{abstract}

Keywords: Mucinous Adenocarcinoma, Cutaneous Metastases, Colon

\section{Introduction}

The general incidence of cutaneous metastasis (CM) of gastrointestinal neoplasms is $5.3 \%{ }^{1}$, and frequency of skin metastasis of colorectal carcinoma has been reported to be 2.3-6\% $\%^{2}$, most of them appearing near the site of the primary tumor. Therefore, the CM of colonic tumors is usually in the pelvis and abdomen ${ }^{3}$. Skin metastases from colorectal carcinoma are rare and typically signify widespread disease with poor prognosis ${ }^{4}$. The most frequent site of cutaneous metastases of colonic cancer is the abdominal skin, often on surgical incision scars ${ }^{5}$. Ulceration, nodules, bullae or fibrotic processes are the most common presentations of cutaneous metastases ${ }^{6}$.

\section{Case Report}

A 55years old man presented with multiple skin nodules on the abdominal wall (Fig.1). These were varying in size from $0.5 \mathrm{~cm}$ to $3 \mathrm{~cm}$ and were firm, mobile and painless. These were clinically thought to be cutaneous metastases.

In another case, a 57 years old lady presented with skin nodules on anterior abdominal wall, varying in size from $0.5 \mathrm{~cm}$ to $2 \mathrm{~cm}$ which were firm, mobile and painless, and thought to be cutaneous metastases.

FNAC was done from the skin nodules. Giemsa stained slides were prepared and Liquid Based Cytology(LBC) was done. LBC slides were stained with Pap stain. Immunocytochemistry was done on FNAC and LBC preparation.
Smears of both the cases showed clusters and sheets of atypical cells with moderate amount of cytoplasm against a necrotic background. The cells showed moderate pleomorphism. Nuclei were round to oval with coarse chromatin and prominent nucleoli. Well formed glandular structures were seen in large aggregates as well as lying separately (Fig. 2).

Immunocytochemistry showed that cells were positive for Muc-1, and negative for vimentin and LCA (Fig. 3,4,5).

Based on these findings, a provisional diagnosis of adenocarcinoma presenting as cutaneous metastasis was given. On detailed history, it was found that both the patients were known cases of carcinoma colon post chemotherapy and radiotherapy. Pre-operative USG in the case of the 57 years old lady showed circumferential bowel wall thickening in right para-umbilical region. Pre-operative PET-CT showed mucosal thickening along hepatic flexure and proximal transverse colon with resultant partial luminal compromise with metastatic deposits in adjascent lymph nodes, omental deposits, and anterior abdominal wall deposits along anterior abdominal wall. Post-operative biopsy of bowel wall of both cases showed mucinous adenocarcinoma invading the muscularis propria (fig. 6)

\section{Discussion}

Skin metastases from colorectal adenocarcinoma generally occur within the first two years after resection of the primary tumor and often present simultaneously with metastases to 


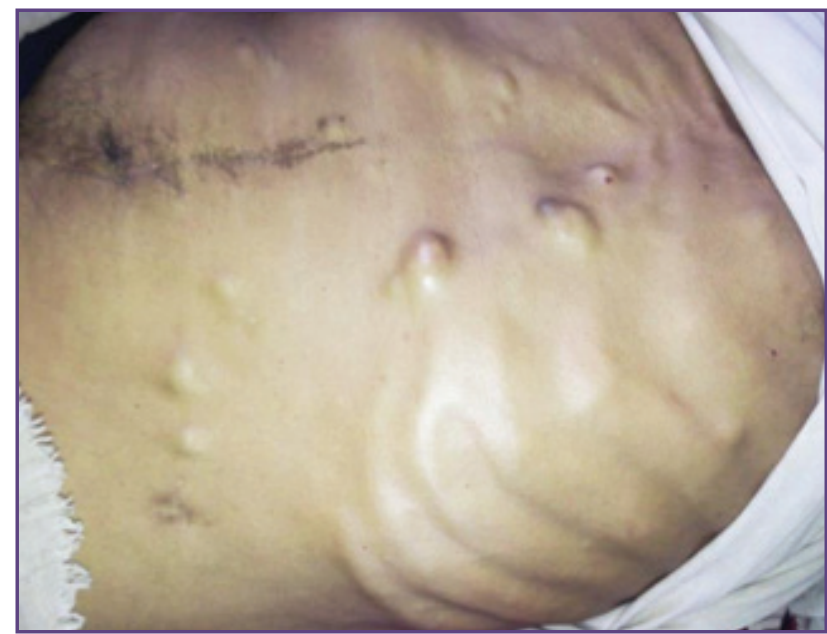

Fig. 1: Multiple skin nodules on abdomen.

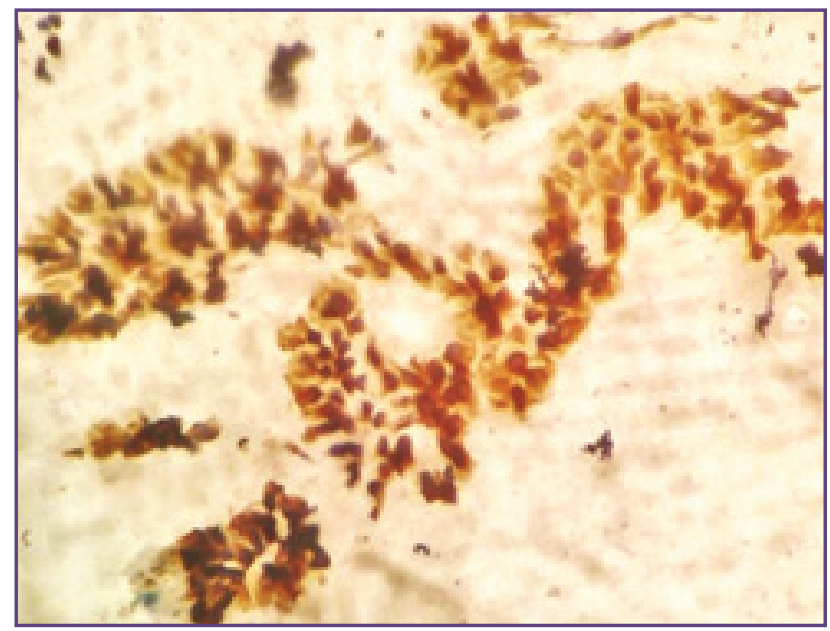

Fig. 3: Positive ICC for Muc-1 (x40).

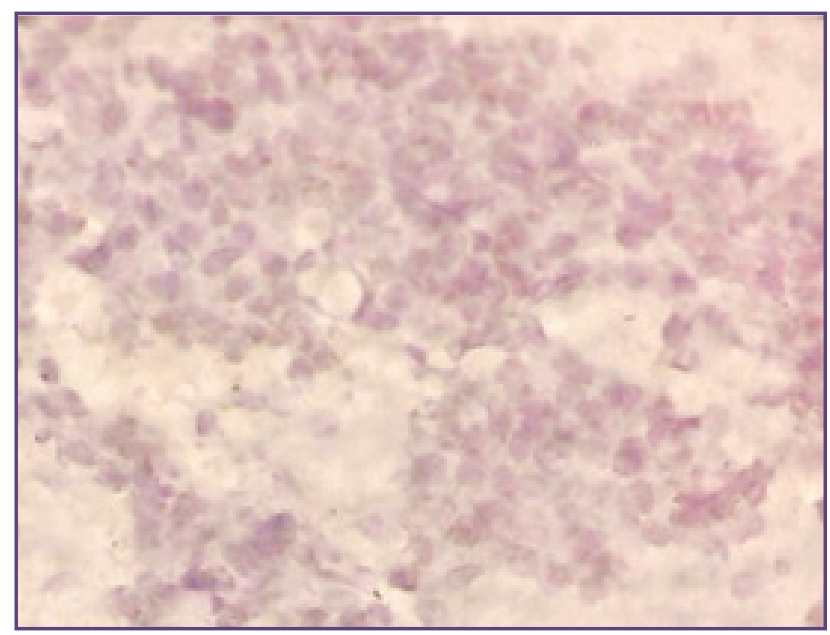

Fig. 5: Negative ICC for LCA (x40).

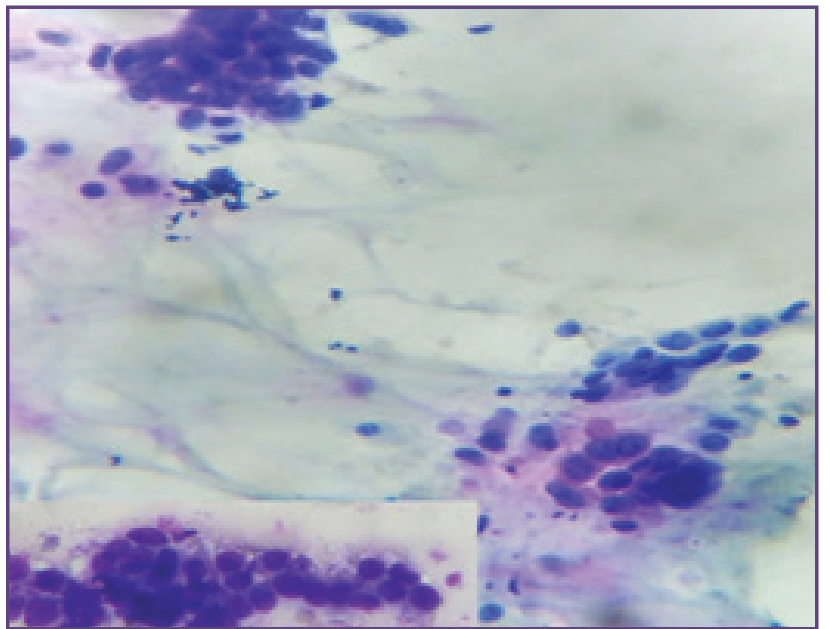

Fig. 2: FNA showing well formed glandular structures (Giemsa x40).

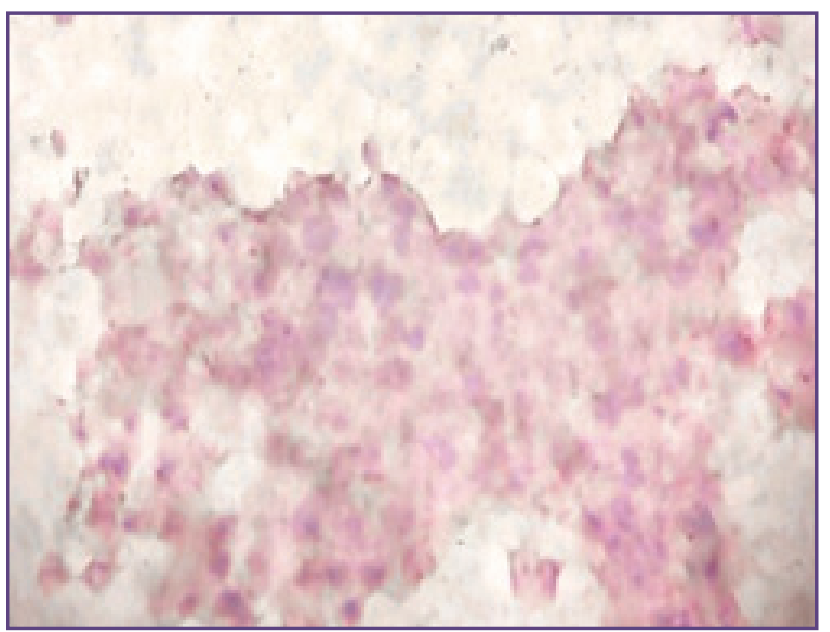

Fig. 4: Negative ICC for Vim (x40).

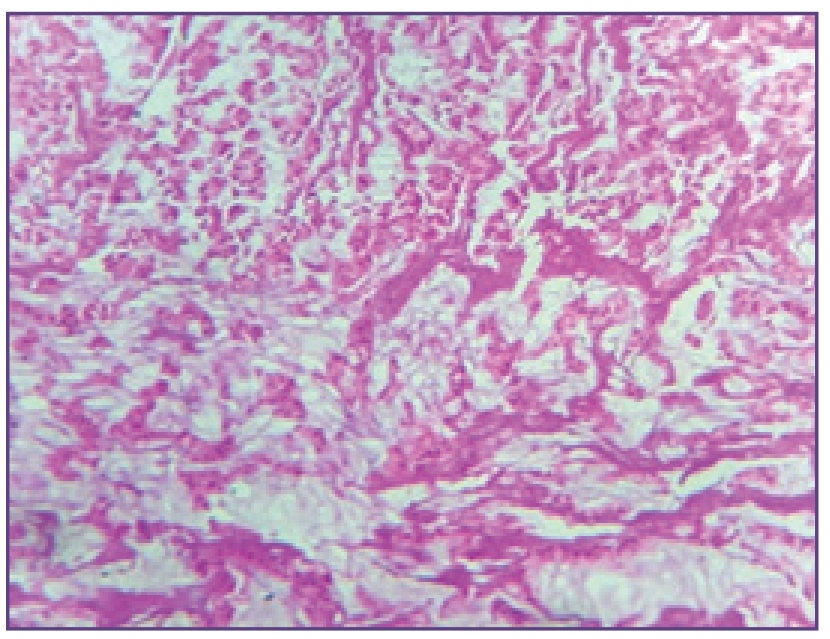

Fig. 6: Biopsy showing well formed glandular (H\&E, x40). 
the liver, peritoneum and lung ${ }^{4}$. The most frequent site of cutaneous metastasis of colonic cancer is the abdominal skin, often on surgical incision scars. This is not exclusive to the ad hoc surgical incision scar, but also to preexisting unrelated operative scars ${ }^{5}$. Other cutaneous sites in order of decreasing frequency are the pelvis, back, chest, upper extremities, head and neck. Uncommon reported sites of skin metastasis of colorectal cancer include scalp, face, eyelids, tip of the nose, nostril, forearm, genitalia, ankle and big toe ${ }^{4}$. Umbilical metastasis (Sister Mary Joseph's nodule) is a recurrent finding ${ }^{5}$.

Cutaneous metastasis may occur through lymphogenous spread, intravascular dissemination, direct extension of tumor and surgical implantation ${ }^{7}$. Wong et al added the spread along embryonal remnants such as the urachus to the aforementioned mechanisms.

Metastatic carcinoma can assume a variety of morphologic appearances ${ }^{7}$. It usually presents as violaceous to fleshcolored, firm, freely mobile, painless nodules, single or multiple. It can sometimes mimic epidermal cysts, neurofibromas, lipomas, cicatricial morphea-like plaques, lymphoma and alopecia ${ }^{7}$. More rarely it can mimic infection, and present as a zone of pink to deep red or purplish red indurated erythema with a well-demarcated border, a condition termed inflammatory metastatic carcinoma or carcinoma erysipelatoides.

Skin metastases can be broadly classified as adenocarcinoma, squamous carcinoma, undifferentiated carcinoma and other types ${ }^{5}$. Most skin metastases from large intestine tumors are well differentiated, often mucinsecreting adenocarcinomas. They usually have a nodular configuration and are located in the dermis, with subsequent spread to the epidermis and subcutaneous tissue.

Identification of skin metastasis from an internal malignancy is considered a poor prognostic sign, as it reflects widespread disease. Survival after diagnosis of cutaneous metastasis ranges from 1 to 34 months. The average survival of patients after diagnosis of skin metastasis from colon cancer is, according to Lookingbill et al., 18 months ${ }^{5}$.

\section{Conclusion}

Colorectal carcinoma presenting as cutaneous metastases is rare and FNAC is a noninvasive and fast method for directing towards the diagnosis of cutaneous metastasis in the patients with a known malignancy without any complication.

\section{References}

1. Krathen RA, Orengo IF, Rosen T. Cutaneous metastasis: a meta-analysis of data. South Med J. 2003; 96:164-7.

2. Pereira WA, Humaire CR, Silva CS, Fernandes LH. Sister Mary Joseph's nodule: a sign of internal malignancy. An Bras Dermatol. 2011;86:S118-S120.

3. Helm TN, Lee TC. Metastatic carcinoma of the skin: treatment \& medication [serial on the Internet] 2010 [cited 2010 Dez 18]. Available from: URL: http://emedicine. medscape.com/Article/1101058-Treatment.

4. Verardino GC, Silva RS, Obadia DL, Gripp AC, Alves Mde F. Rare cutaneous metastasis from a probable basaloid carcinoma of the colon mimicking pyogenic granuloma. An Bras Dermatol. 2011;86:537-540.

5. Krathen RA, Orengo IF, Rosen T. Cutaneous metastasis: A meta-analysis of data. South Med J. 2003;96:164-166.

6. Lo Russo G, Accarpio F, Spinelli G, Miele E, Borrini F, Cerbone L et al. Subcutaneous metastases from colon cancer: a case report. Journal of Medical Case Reports. 2012; 6:212

7. $\mathrm{Gu}$ Y, Tang R, Gong DQ, Qian YL. Reconstruction of the abdominal wall by using a combination of the human acellular dermal matrix implant and an interpositional omentum flap after extensive tumor resection in patients with abdominal wall neoplasm: a preliminary result. World J Gastroenterol. 2008;14:752-757.

*Corresponding author:

Dr. Gireesha Rawal, Department of Pathology, Vardhman Mahavir Medical College and Safdarjung Hospital, New. Delhi. INDIA

E-mail: gireesharawal@yahoo.in,

Date of Submission : 11.09.2016

Date of Acceptance : 24.11.2016

Financial or other Competing Interests: None.

Date of Publication : 19.02.2017 